\title{
Tuberculous Sacroiliitis with Secondary Psoas Abscess: A Case Report
}

\author{
Kaba Condé1,2, Carlos Othon Guelngar'1, Igué Kadidjatou1, D. D. Granga 3, Mamadou Ciré Barry, \\ Mamadou Hady1, Karinka Diawara1, Konaté Mamady², Touré Moriba², Awada Mohamed², \\ Fodé Abass Cissé ${ }^{1}$
}

\footnotetext{
${ }^{1}$ Department of Neurology, Academic Hospital, University of Conakry, Conakry, Guinea

${ }^{2}$ Department of Rheumatology, Academic Hospital, University of Conakry, Conakry, Guinea

${ }^{3}$ Department of Pediatric, Mother and child Hospital, Ndjamena, Chad

${ }^{4}$ Department of Pediatric, Academic Hospital, University of Conakry, Conakry, Guinea

Email: Condekba95@yahoo.fr, car1325@yahoo.fr, awadamohamed2@gmail.com,kadigue95@gmail.com, drbarrymckadi08@yahoo.fr, hadyd08@gmail.com,diawarakarinka@yahoo.fr,medkonate90@gmail.com, touremoriba@yahoo.fr,dounag@yahoo.fr, abass3002yahoo.fr
}

How to cite this paper: Condé, K., Guelngar, C.O., Kadidjatou, I., Granga, D.D., Barry, M.C., Hady, M., Diawara, K., Mamady, K., Moriba, T., Mohamed, A. and Cissé, F.A. (2021) Tuberculous Sacroiliitis with Secondary Psoas Abscess: A Case Report. Open Journal of Rheumatology and Autoimmune Diseases, 11, 48-52. https://doi.org/10.4236/ojra.2021.112006

Received: December 8, 2020

Accepted: April 24, 2021

Published: April 27, 2021

Copyright ( 2021 by author(s) and Scientific Research Publishing Inc. This work is licensed under the Creative Commons Attribution International License (CC BY 4.0).

http://creativecommons.org/licenses/by/4.0/

\section{(c) (i) Open Access}

\begin{abstract}
Tuberculous sacroiliitis secondary to a psoas abscess is rare, only a few sporadic cases were reported in the literature. Tuberculous sacroiliitis is rare, usually unilateral, its symptomatology is misleading, its diagnosis is often delayed or even confused with damage to the hip or lumbosacral hinge, most often related to difficulties exploration of the sacroiliac joint. We report the case of a 66-year-old diabetic patient with low back pain, unilateral right with inflammatory appearance, insidious installation, evolving for about 8 months. The diagnosis of tuberculous sacroiliitis was made after biopsy of the sacroiliac joint. CT and MRI are necessary for lesion diagnosis. Tuberculosis treatment was started and the abscess was surgically drained. The aim of this work was to describe the diagnostic pathway of a patient with tuberculous sacroiliitis in a tropical environment. Conclusion: Tuberculous sacroiliitis, secondary to an abscess of the psoas muscle is an unusual cause of hip pain and is likely to be overlooked due to its atypical presentation.
\end{abstract}

\section{Keywords}

Tuberculous Sacroiliitis, Psoas Abscess, Guinea

\section{Introduction}

Osteoarticular tuberculosis is uncommon, estimated at $1 \%$ to $3 \%$ of extra-pulmonary tuberculosis [1]. Tuberculous sacroiliitis is rare, usually unilateral, its 
symptomatology is misleading [2], its diagnosis is often delayed or even confused with damage to the hip or lumbosacral hinge, most often related to difficulties exploration of the sacroiliac joint [3] [4]. We report a case of tuberculous sacroiliitis secondary to an abscess of the psoas muscle.

\section{Observation}

\section{Patient's Consent}

A 66-year-old patient with a history of blood hypertension and diabetes, hospitalized for lumbar-fessalgia, right unilateral inflammatory appearance, insidious installation, evolving for about 8 months. He had been treated with Coversyl, Metformin and Tramadol. He describes neither fever, nor the concept of contagion, nor respiratory symptoms, or weight loss. On physical examination, the patient was in good general condition. There was a pain on the pressure of the spinous processes. The Schöber index and Lasegue's sign were impossible due to the pain. The sacroiliac pressure was painful, Ericksen's and Volkmann's manoeuvres were positive. There was no lymphadenopathy. The remainder of the physical examination was unremarkable.

The biological assessment found a normal blood count, a non-specific biological inflammatory syndrome with an erythrocyte sedimentation rate (ESR) accelerated to $86 \mathrm{~mm}$ at the 1st hour, a positive C-reactive protein (CRP) at 59.3 $\mathrm{mg} / \mathrm{l}$. The hepatic and renal function was normal. The HLA-B27 antigen was immunologically negative. Blood cultures were positive on two occasions. The human immunodeficiency virus (HIV) serology was negative.

The standard X-ray of the lumbar spine and pelvis was unremarkable; however, CT scan of the pelvis (Figure 1(a)) showed severe right unilateral sacroiliitis with intra-auricular effusion (Forest stage III). Lumbar (Figure 2) and pelvis

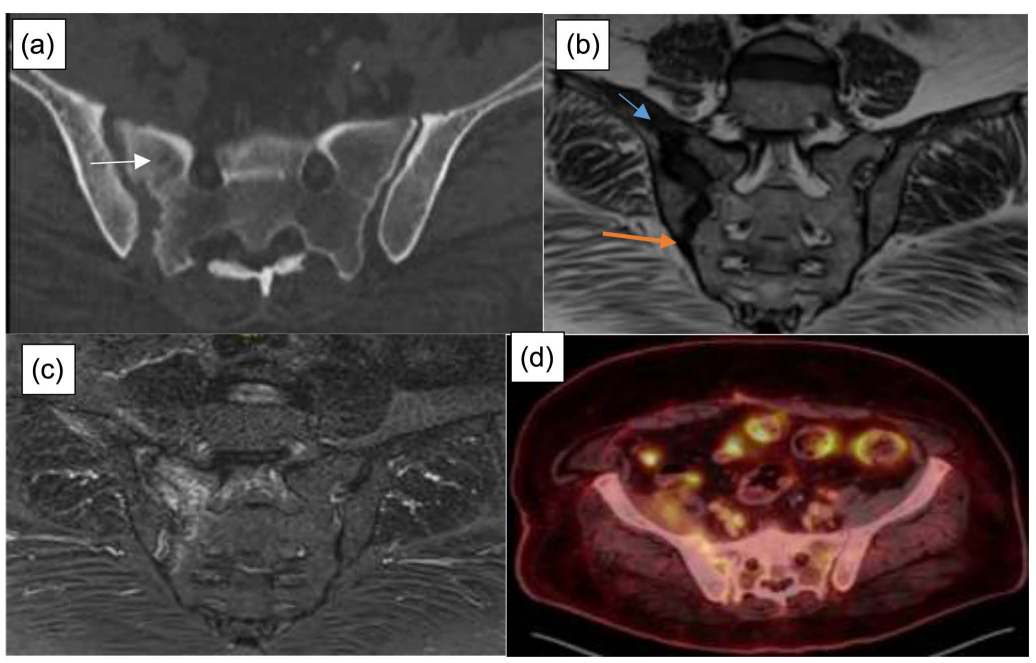

Figure 1. (a) CT scan of the pelvis showing right sacroiliitis. Sacroiliac MRI ((b) sequence T1 and (c) sequence T2) showing right sacroiliitis (white arrow) with intra-articular effusion and infiltration of the psoas muscle (blue arrow); (d) PET scan showing heterogeneous hypermetabolism of the psoas muscle and the right sacroiliac joint (blue arrow). 


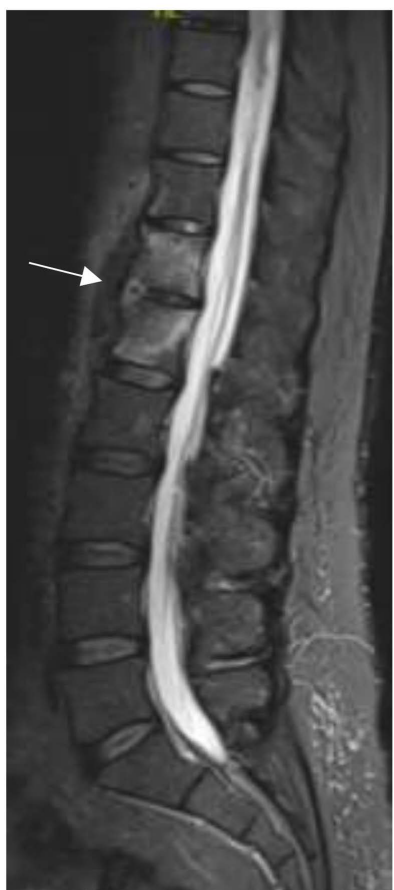

(a)

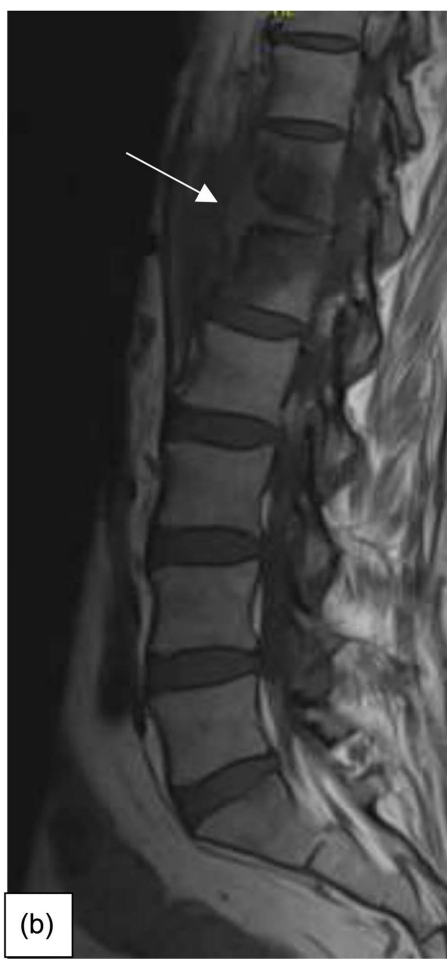

(b)

Figure 2. Lumbar MRI scan ((a) sequence T1 and (b) sequence T2) showing a D12-L1 spondylodiscitis with infiltration of the pre-vertebral space.

(Figure 1(b) and Figure 1(c)) MRI scar further revealed D12-L1 spondylodiscitis with infiltration of the pre-vertebral space and the right psoas muscle. The PET scan noted heterogeneous hypermetabolism of the psoas muscle and the right sacroiliac joint (Figure 1(d)). Drainage of the abscess and a fluoroscopic biopsy of the right sacroiliac joint were positive for mycobacterium tuberculosis. The chest X-ray was normal while the tuberculin intradermal reaction was positive at $15 \mathrm{~mm}$. The diagnosis of tuberculous sacroiliitis secondary to a psoas abscess was retained. Quadruple therapy combining isoniazid, rifampicin, pyrazinamide and ethambutol was started for 12 months and the abscess was surgically drained. After a follow-up of 6 months, all the clinical symptoms regressed and the laboratory parameters returned to normal.

\section{Discussion}

The incidence rate of tuberculosis continues to decline worldwide [3], but it remains a major public health concern in developing countries due to the AIDS pandemic [5]. Osteoarticular tuberculosis accounts for 3\% of extrapulmonary tuberculosis, of which $5 \%-8 \%$ concerns the sacroiliac joint [1] [2] [6]. Tuberculous sacroiliitis secondary to psoas abscess is rare, only a few sporadic cases have been reported in the literature [3] [5] [6]. A predisposing risk factor is sometimes found HIV infection, postpartum, transplantation, ulcerative colitis, prolonged corticosteroid therapy [7]. In our case, the only risk factor found was diabetes. 
In Africa these are short series and occasional observations that have been reported as in the Maghreb especially in Tunisia or Bouajina et al. [8] reported 22 cases and tuberculous sacroiliitis as well as in Sub-Saharan Africa, in Burkina Fasso [5] have reported 2 cases of tuberculous sacroiliitis. However, there was no associated psoas abscess as reported in observation.

Tuberculous sacroiliitis is insidious, the time between the onset of clinical signs and hospitalization is long (approximatively 2 weeks to 6 months) [9]. It is characterized by the predominance of local symptoms as demonstrated in this case. The patient had no systemic symptoms and pain is the most common complaint. The diagnosis of osteoarticular tuberculosis is based on the culture of biopsy material, sputum is negative except in the very rare cases with concomitant pulmonary tuberculosis were reported [3] [9]. The main species identified is mycobacterium tuberculosis [3].

The radiological lesions of sacroiliitis are very progressive onset. Tuberculous arthritis is very destructive, most often giving images of erosion and geodes [9]. CT scan appears to be the test of choice for the detection of sequesters, while MRI is more sensitive for the detection of early cancellous bone abnormalities as well as abscesses regardless of their size [10].

The introduction of anti-tuberculosis chemotherapy resulted in a dramatic improvement in the prognosis. Psoas abscess is treated with antibiotic therapy combined with drainage [11]. However, tuberculosis abscesses often heal with anti-tuberculosis treatments without drainage [2] [11]. The duration of treatment for osteoarticular tuberculosis should not be less than 12 months or even extended to 18 months [12].

\section{Conclusion}

Tuberculous sacroiliitis is rare and its clinical presentation is atypical. The diagnosis must be made in the event of low back pain and/or pain in the hip. Our case confirms the rarity and the non-specific nature of the clinical manifestations of tuberculous sacroiliitis, generally leading to a delay in diagnosis. The CT scan, MRI and biopsy are necessary for diagnosis.

\section{Conflicts of Interest}

The authors declare that they have no conflicts of interest.

\section{References}

[1] Sahu, R. (2011) Turner Syndrome with Tubercular Osteomyelitis of Iliac Bone: An Unusual Presentation. Indian Health Journal. https://www.researchgate.net/publication/261216229_TUBERCULOSE_DE_L\%27 OS_ILIAQUE_UNE_LOCALISATION_INHABITUELLE

[2] Prakash, J. (2014) Sacroiliac Tuberculosis-A Neglected Differential in Refractory Low Back Pain-Our Series of 35 Patients. Journal of Clinical Orthopaedics and Trauma, 5, 146-153. https://doi.org/10.1016/j.jcot.2014.07.008

[3] Kramer, L., Geib, V., Evison, J., Altpeter, E., Basedow, J. and Brügger, J. (2018) Tu- 
berculous Sacroiliitis with Secondary Psoas Abscess in an Older Patient: A Case Report. Journal of Medical Case Reports, 18, 237. https://doi.org/10.1186/s13256-018-1754-4

[4] Özdemir, Z.M., Kahraman, A.S., Görmeli, C.A., Sevimli, R. and Akpolat, N. (2016) Langerhans Cell Histiocytosis with Atypical Intervertebral Disc and Sacroiliac Joint Involvement Mimicking Osteoarticular Tuberculosis in an Adult. Balkan Medical Journal, 33, 573-577. https://doi.org/10.5152/balkanmedj.2016.160492

[5] Diallo, I., Zabsonré, J.T., Tiemtoré Kambou, B.M.A., Sondo, A.K., Sagna, Y. and Ouédraogo, D.D. (2016) Sacroillite Tuberculeuse: A Propos de deux cas. Pan African Medical Journal, 25, 69.

[6] Shields, D.W. and Robinson, P.G. (2012) Iliopsoas Abscess Masquerading as 'Sciatica'. BMJ Case Reports, 2012. https://doi.org/10.1136/bcr-2012-007419

[7] Nair, K.R. and Jayachandran, R. (2013) Postpartum Tuberculous Sacroiliitis. American Journal of Orthopedics (Belle Mead, N.J.), 42, 16-17.

[8] Bouajina, E., Harzallah, L., Hachfi, W., Bel Hadj Slama, K., Rammeh, N., Ghannouchi, M., Bahri, M. and Letaief, A. (2005) Sacro-Iliites Tuberculeuses : A propos de 22 cas. La revue de médecine interne, 26, 690-694.

[9] Ali Chaudhry, L. and Al-Solaiman, S. (2013) Multifocal Tuberculosis: Many Faces of an Old Menace. The International Journal of Mycobacteriology, 2, 58-69. https://doi.org/10.1016/j.ijmyco.2013.01.001

[10] Albano, D., Treglia, G., Desenzani, P. and Bertagna, F. (2017) Incidental Unilateral Tuberculous Sacroiliitis Detected by 18F-FDG PET/CT in a Patient with Abdominal Tuberculosis. Asia Oceania Journal of Nuclear Medicine and Biology, 5, 144-147.

[11] Navarro López, V. (2015) Worldwide Variations over the Years in Etiology of Iliopsoas Abscess. Reality or a Selection Bias? Medicina Clínica (Barc), 144, 259-260.

[12] WHO (2010) Treatment of Tuberculosis: Guidelines. http://www.who.int/tb/publications/2010/9789241547833/en/ 